\title{
Management of oral and maxillofacial infection. where shall we start?
}

\section{Editorial}

Management of the oral and maxillofacial infection is not an informal procedure as many believe. The concept of infection management mean antibiotic prescription is totally wrong. The rationale behind management of the oral and maxillofacial infection is eradication of the infection which can't achieved with just antibiotic prescription. In this editorial we mentioned general rules for management of oral and maxillofacial infection. We stared with rule number 2 and ended with rule number. ${ }^{1}$

Rule number 2: Don't underestimate the power of infection: Oral and maxillofacial infection is a daily case in the dental clinic with serenity range from periapical abscess to infection of the fascial space. Deep neck space infections can spread along the fascial spaces of the head and neck, inducing life-threatening deep space infection associated with a high risk of complications. The morbidity and mortality of oral and maxillofacial infection ranging from airway obstruction to cerebral abscess, Lemierre's syndrome and death. Most of the complications resulted from delaying or inaccurate inference. Dentists should be always alert to the potential complication of the infection that can be life threating complication. Rapid deterioration of the patient with odontogenic infection can be easily happed especially in immune-compromised patients (e.g. Diabetic patients).

Rule number 3: Know the enemy and know yourself (Sun Tzu, The Art of War): The knowledge of the micro-organisms causing the odontogenic infection is the key-stone in the management of the infection. Incorrect selection of the antibiotic adding no benefits for the patient but worsen the case. Even the application of the empirical antibiotic should be based on sound scientific bases. The odontogenic infections are mixed aerobic-anaerobic infections. The aerobic bacteria represent $25 \%$ of the total number of the bacteria causing the odontogenic infection while anaerobic represent $75 \%$. The combination of amoxicillin/clavulanate and metronidazole is a widely-accepted empirical regimen for infections of the odontogenic spaces.

Rule number 4: Trait the anatomy: A detailed knowledge of the fascial planes and anatomical routes of spread of the infection is a critical requirement to understanding the clinical manifestations, potential complications, and appropriate management of these infections. The fascia plays an important role in determining the location and course of spread of the odontogenic infections. Prediction of the possible fascial space involvement by the odontogenic infection and the subsequent complication is anatomy based knowledge.

Rule number 5: Listening: Taking patient History (the patient story) is an important step in the diagnosis of odontogenic infection. The dentist does not only treat "teeth in patients "but patients who have teeth or not". The patient can tell a word to the dentist which will be a guide to the correct diagnosis of the cause of the infection. So; careful history taking with analysis of the patient story is an important role in diagnosis of the cause of the infection.
Volume 7 Issue 3 - 2017

Ayman Hegab
Al-Azhar University, Egypt

Correspondence: Ayman Hegab, Clinical Associate Professor of Oral \& Maxillofacial Surgery,Al-Azhar University, Cairo, Egypt, Email hegab@mail.com

Received: May 20, 2017 | Published: May 24, 2017

Rule number 6: Examination of the patient: with the advanced technology in the imaging studies, radiographic evaluations became an essential part in the patient examination. Contract- enhanced CT and MRI are now commonly used for the diagnosis of the infection and its spreading route. Treatment plan for patient with deep space infection mainly based on examination and detection of the involved spaces for adequate drainage.

Rule number 7: removal of the etiologic factor: one shouldn't miss the etiology of the infection. Eradication of the infection can be achieved through removal of the etiologic factor either by root canal treatment or extraction of the causative tooth or teeth. Persistence of the etiologic tooth or teeth represents failure of the treatment with possibility of recurrence of the infection.

Rule number 8: Antibiotic therapy and incision and drainage: antibiotic therapy is an adjunctive therapy and not an alternative to dental intervention. Drainage of infection can be achieved through the canal by the root canal treatment or through the socket by extraction of the tooth. Intraoral and extraoral incisions are required for adequate drainage of the fascial space. The art and science of using the antibiotic is the key of success to prevent the Antibiotic apocalypse. The application of the principle of Antimicrobial Stewardship is important to optimize the clinical outcomes while minimizing unintended consequences of antimicrobial use. Antimicrobial Stewardship consisted of 4 rights, using the right antibiotic at the right time at the right dose for the right duration.

Rule number 1: Timing: Timing is the most important factor in the management of odontogenic infection to prevent the subsequent airway complication. In 1974, Dr. Guralnick applied the principles of the initial establishment of airway security, followed by early and aggressive surgical drainage of all anatomic spaces affected by cellulitis or abscess under adequate antibiotic control. Severity score of the fascial space infections is important to know the risk of airway involvement by the infection. The score range from 1 to 4 . 
Severity score 1: which include fascial spaces with low risk to airway or vital structures. The fascial spaces are vestibular, subperiosteal, infraorbital, and buccal spaces.

Severity score 2: which include fascial spaces with moderate risk to airway or vital structures. The fascial spaces are submandibular, submental, sublingual, pterygomandibular, submassteric, superficial and deep temporal spaces.

Severity score3: which include fascial spaces with high risk to airway or vital structures. The fascial spaces are lateral pharyngeal, retropharyngeal, and pretracheal spaces.

Severity score 4: which include fascial spaces with extreme risk to airway or vital structures. The fascial spaces are Danger space (space 4), mediastinum, and intracranial infection.

\section{Funding}

None.

\section{Acknowledgments}

None.

\section{Conflicts of interest}

The author declares that there is no conflict of interest.

\section{References}

1. Miloro M, Ghali GE, Larsen P, et al. Peterson's Principles of Oral and Maxillofacial Surgery. 3rd ed. Connecticut, USA: People's Medical Publishing House; 2012. 841-861 p. 